\title{
Age- and Sex-Related Variations in the Brain White Matter Fractal Dimension Throughout Adulthood: An MRI Study
}

\author{
S. Farahibozorg • S. M. Hashemi-Golpayegani · \\ J. Ashburner
}

Received: 22 June 2013 / Accepted: 27 November 2013 / Published online: 12 January 2014

(C) The Author(s) 2013. This article is published with open access at Springerlink.com

\begin{abstract}
Purpose To observe age- and sex-related differences in the complexity of the global and hemispheric white matter (WM) throughout adulthood by means of fractal dimension (FD).

Methods A box-counting algorithm was used to extract FD from the WM magnetic resonance images of 209 healthy adults from three structural layers, including general ( $\mathrm{gFD}$ ), skeleton (sFD), and boundaries (bFD). Model selection algorithms and statistical analyses, respectively, were used to examine the patterns and significance of the changes.

Results gFD and sFD showed inverse U-shape patterns with aging, with a slighter slope of increase from young to mid-age and a steeper decrease to the old. bFD was less affected by age. Sex differences were evident, specifically in gFD and sFD, with men showing higher FDs. Age $\times$ sex interaction was significant mainly in the hemispheric analysis, with men undergoing sharper age-related changes. After adjusting for the volume effect, age-related results remained approximately the same, but sex differences changed in most of the features, with women indicating higher values, specifically in the left hemisphere and boundaries. Right hemisphere was still more complex in men.
\end{abstract}

S. Farahibozorg $(\bowtie) \cdot$ S. M. Hashemi-Golpayegani Department of Biomedical Engineering,

Amirkabir University of Technology, 424, Hafez Ave.,

Tehran 1591634311, Iran

e-mail: r-farahi@aut.ac.ir

J. Ashburner

Wellcome Trust Centre for Neuroimaging,

Institute of Neurology, University College London,

12 Queen Square, WC1N 3BG, London, UK
Conclusions This study is the first that investigates the WM FD spanning adulthood, treating age both as a continuous and categorical variable. We found positive correlations between FD and volume, and our results show similarities with those investigating small-world properties of the brain networks, as well as those of functional complexity and WM integrity. These suggest that FD could yield a highly compact description of the structural changes and also might inform us about functional and cognitive variations.

Keywords Fractal dimension - Complexity · Aging brain $\cdot$ Sex difference $\cdot$ Brain MRI $\cdot$ White matter

\section{Introduction}

Normal aging is associated with functional and structural changes in the human brain. In recent decades, and particularly since the advent of magnetic resonance imaging (MRI) scanners, many studies have been conducted to detect these alterations. White matter (WM), as one of the two brain tissues, mainly consists of glial cells and myelinated axons and undergoes several changes in normal aging. The studies focused on changes occurring in the WM structure in normal adults from MR images can broadly be categorized into two groups of volumetric analysis and shape analysis. For the first group, a discrepancy is evident among their results: while most have found shrinkage of global WM [1], some have reported no significant difference with aging [2]. Second group, investigating geometrical changes, which include, but are not restricted to, WM hyperintensities (WMH) in T2-weighted images [3], lower magnetic transfer ratio (MTR) [3], lower fractional anisotropy (FA) in diffusion tensor images (DTIs) [3-5], and changes in 
structural networks [6] and connectivity [4]. These groups of changes are usually due to the loss of myelin sheets and axonal fibers resulting in a reduction in the WM integrity [3]. Changes of the complexity of the brain WM are also investigated in a few studies [7], but these are not prevalent due to methodological challenges [8]. Structural complexity may provide a compact description of a group of structural changes [9]. Thus, in the current study, we have focused on the brain structural complexity by means of fractal dimension (FD).

FD, introduced by Mandelbrot [10], is a substitute for Euclidean dimension in the case of complex objects that indicate self-similarity, which is applicable to many natural phenomena. For non-Euclidean objects, FD usually exceeds topological dimension and is noninteger, whereas for ordinary shapes, it is equal to the traditional Euclidean dimension. Therefore, FD as an index of statistical complexity has been used in several fields of study, including medicine and biology [11-14]. We chose FD for this study because it is compatible with the geometry of the brain and can serve as a structural signature by compressing many characteristics into a single number [9]. Moreover, as reported by some previous studies [9-15], there is a positive correlation between FD of the gray matter (GM) and WM and one's intelligence and cognitive performance. Therefore, our results, in addition to quantifying structural changes, could also account for age- and sex-related differences in the brain and cognitive abilities.

FD has previously been used to quantify the changes occurring in the complexity of the brain in some diseases, as well as in normal aging. In [7], the authors extracted this feature from brain MR images to compare WM complexity between young and older normal adults. They found that WM complexity significantly decreases with aging, and this change was specifically seen in the WM skeleton. They also reported gender differences, with men showing higher FDs than women, and age-related effects were more visible in the left hemisphere for men, but in the right hemisphere for women. In this study, we pursue an analogous aim, namely the investigation of age- and sex-related changes in the FD of three structural layers during adulthood. The dataset we use is a larger sample covering a substantial age range of adult life (20-80 years). It allows age to be treated as a continuous variable, and also enables comparisons of three groups of young, middle age, and old instead of the usual two. We also investigate the correlations between WM volume and FD and the effects of the former on the latter in detail. These could shed more light on the clinical significance and applicability of FD.

\section{Materials and Methods}

\section{Subjects}

The data used for this study are a part of the publicly accessible Information eXtraction from Images (IXI) database [16]. The whole dataset contained images from three scanners, and subjects were divided into five ethnic groups and five levels of qualification. To reduce the variations and nonnormality and obtain more precise results from the comparative analysis, we only used the data gathered from one of the scanners, a Philips 1.5-T system at Guy's Hospital, and limited our analysis to the subjects of white ethnicity and with a high school diploma or higher education. Hence, our data contain brain MR images of 209 psychologically normal subjects aged 20-80 (49.31 \pm 15.5$)$ years, among which 95 are men (age: $47.54 \pm 15.2$ years) and 114 are women (age: $50.78 \pm 15.7$ years). We first investigated age as a continuous variable, then also categorized subjects into three groups of young, mid-age, and old, labeling subjects aged less than 40 years as young ( 36 men aged $31.71 \pm 5.2$ years and 31 women aged $30.1 \pm 5.5$ years), those aged between 40 and 60 years as mid-age ( 35 men aged $50.19 \pm 6.6$ years and 48 women aged $51.58 \pm 6.5$ years), and those aged more than 60 years as old ( 24 men aged $67.42 \pm 5.1$ years and 35 women aged $68.02 \pm 5.8$ years). This categorization might provide an opportunity for more convenient comparisons between our results and those of previous studies, which have considered age-groups.

\section{Image Acquisition}

The T1-weighted MR images had been acquired on a Philips Gyroscan Intera 1.5-T scanner at Guy's Hospital, with repetition time $\sim 9.81 \mathrm{~ms}$, echo time $\sim 4.6 \mathrm{~ms}$, number of phaseencoding steps $=192$, echo train length $=0$, reconstruction diameter $=240 \mathrm{~mm}$, and flip angle $=8$. For each image, the original pixel size was approximately $0.938 \times 0.938 \times 1.2 \mathrm{~mm}$, and the matrix representing each sagittal plane was $256 \times 256$, with 150 planes for each subject.

\section{Preprocessing}

All preprocessing and postprocessing of the MR images, as well as modeling and statistical analysis, are performed using MATLAB R2009a or R2012a (MathWorks, Natick, MA) in this study. For the preprocessing, as illustrated in Fig. 1, the "New Segment" toolbox of the SPM8 package was used to segment the structural scans and extract the WM. The procedure includes an integrated modeling framework, which combines tissue classification, bias correction, and nonlinear warping, altogether [17]. For this purpose, images should be first coarsely aligned with tissue probabil- 


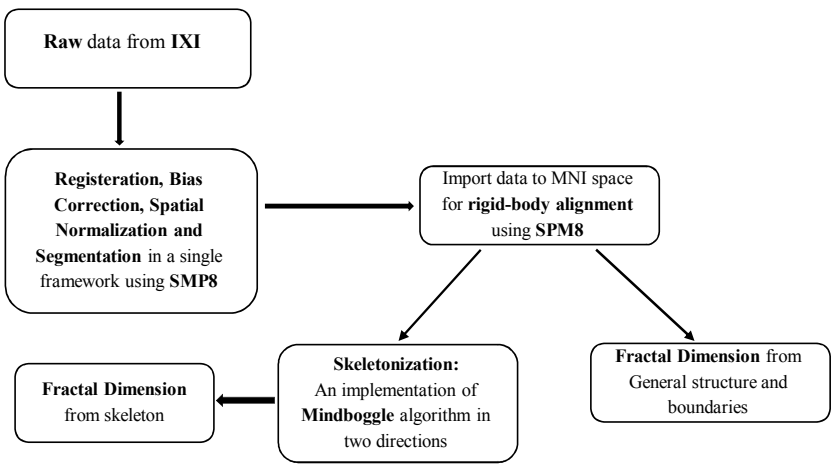

Fig. 1 A flowchart representing steps of the pre- and postprocessing of the magnetic resonance images

ity maps, which are modified versions of ICBM (International Consortium for Brain Mapping) Tissue Probabilistic Atlases. Then, a generative model is used, which includes parameters for tissue classification and registration, as well as parameters for image intensity inconsistencies. To estimate the parameters of this model, an iterative switching among three steps of classification, bias correction, and registration is done to finally result in the desired posterior probabilities for each tissue class.

After segmentation, the images were slightly repositioned using the closest rigid-body transform to roughly match MNI (Montreal Neurological Institute) space and be in rigid-body alignment with each other [18]. They were then down-sampled to a symmetrical voxel size of $1 \times 1 \times 1 \mathrm{~mm}$, yielding images of size $181 \times 217 \times 181$. We used vertical mid-plane of these so-called "imported" images to separate the two hemispheres of the brain.

\section{Skeletonization}

Skeletonization is the procedure of removing as many boundary voxels as possible, without letting the image break apart. Preserving the connections is an important issue in skeletonization algorithms, as the skeletons should keep the topological properties of an image. We used a modification of the algorithm available in the Mindboggle toolbox [19]. The original program runs a pseudo-3D algorithm, which skeletonizes the 3D image plane by plane and then integrates the results from planar images. As in this procedure, the connectivity will be preserved only in one direction, we implemented it in two different directions (coronal and transverse) and obtained the final result as a combination of these two. To obtain a skeleton with fully preserved connections, one might expect skeletons derived from sagittal planes to be merged as well. However, it would be useful to note that implementing the procedure in multiple directions would affect one-voxel thickness of the skeleton, and it might become thick in some regions (as the procedure is done independently in each direction). Therefore, what we have done is a trade-off between obtaining fully connected versus thin skeletonizations. Because the box sizes used to calculate FD (to be explained shortly) were two voxels or larger, slight disconnections would not affect FD significantly. We also tested a fully parallel $3 \mathrm{D}$ algorithm suggested in [20], but it was significantly slower, while thinning and connectivity of the outcome was not ideal either.

\section{Three-Dimensional Fractal Dimension}

Among several approaches available to calculate the FD, the one known as box-counting (BC) [21] is used here. BC is the most frequently used method for fractal analysis of brain images due to its simplicity and robustness. Also, as $\mathrm{BC}$ does not require the objects to be strictly self-similar, it is appropriate for brain analysis. This is because the human brain, like many other natural structures, is self-similar only over particular scales.

The core of all $\mathrm{BC}$ algorithms involves first covering the whole image with a mesh of a minimum box size (r) and counting the number of boxes required to cover the object entirely $(\mathrm{N})$. By repeating the procedure over a range of values of $\mathrm{r}$, the corresponding values for $\mathrm{N}$ are calculated. Figure 2a shows images of the WM original tissue and skeletonized version, each covered with two meshes of box sizes 4 and 8 , respectively. Thereafter, plotting $\log _{\mathrm{e}}(\mathrm{N})$ versus $\log _{\mathrm{e}}(1 / \mathrm{r})$ would result in a diagram similar to Fig. $2 \mathrm{~b}$. Now, if we refer to points within the object as "Black" and those in the background as "White," covering an image with a mesh would give three kinds of boxes: black, white, and gray (boxes that include both object points and background). In traditional BC methods, all boxes that wholly or partially contain object points are counted to result N. However, in this study, we used an algorithm that is analogous to the one introduced for 3D images in [8]. The algorithm defines five different box counts rather than only one: $\mathrm{N}_{\mathrm{B}}$ : black boxes; $\mathrm{N}_{\mathrm{W}}$ : white boxes; $\mathrm{N}_{\mathrm{G}}$ : gray boxes (this represents the boundaries of the object); $\mathrm{N}_{\mathrm{BW}}$ : sum of $\mathrm{N}_{\mathrm{B}}$ and $\mathrm{N}_{\mathrm{W}}$; and $\mathrm{N}_{\mathrm{BG}}$ : sum of $\mathrm{N}_{\mathrm{B}}$ and $\mathrm{N}_{\mathrm{G}}$. As can be understood, $\mathrm{N}_{\mathrm{BG}}$ is equal to $\mathrm{N}$ in traditional $\mathrm{BC}$ methods.

Two major issues in performing $\mathrm{BC}$ are the choice of box size range and the approach used for the single slope analysis of linear area in the logarithmic plot for FD estimation. For the former, box sizes of 2 to one-third of the minimum dimension of the 3D image (which is 60 in this study) were used, as suggested in [8]. For the single slope analysis as well, we followed a same approach as reference [8]. Suppose that we have $K$ different box sizes and corresponding Ns. We divide this point set into pieces of length $L$, where the first segment includes points $1,2, \ldots, L$, second includes $2,3, \ldots, L+1$, and so on. Afterward, a first-order polynomial is fitted to each segment, and the correlation coefficients between the real point set and the fitted one is computed. 

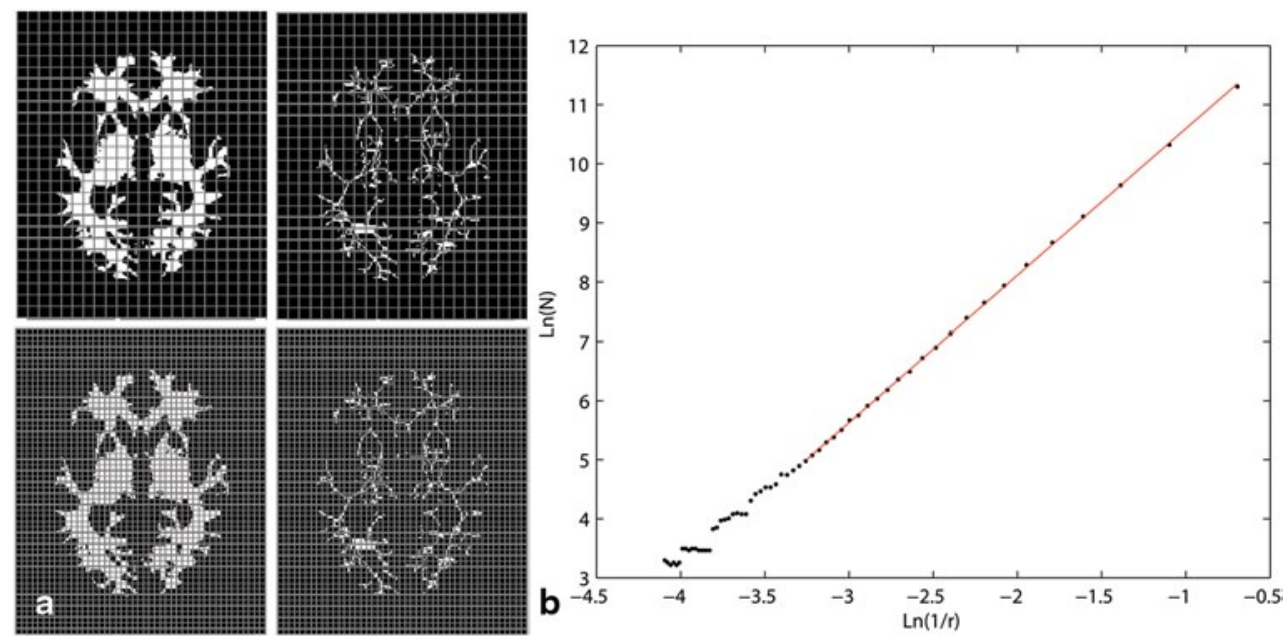

Fig. 2 a original (left) and skeletonized (right) white matter (WM) covered with a mesh with box sizes 8 and 4 (top and bottom, respectively). The numbers of 8 -voxel boxes required to cover the whole original image and the borders were 2,793 and 2,744, respectively, and the number of boxes to cover the skeleton was 2,467. For the 4-voxel boxes, these values were 15,292, 12,934, and 11,627. b A double-logarithmic $(\log -\log )$ plot of the number of boxes required to cover $3 \mathrm{D}$
WM (N) versus the reciprocal of the box length (1/r). Black point set shows measurements at different scales, whereas the red line shows a trend of the linear fit to the point set. In the applied process, the linear fit consisted of a group of segments of length 11, with overlaps (as described in the text). To estimate fractal dimension, the average of the slopes of a group of these segments is calculated, such that the standard deviation of their slopes would not be more than 0.01
The segments are then sorted according to their correlations, and those with the greatest correlations are chosen, until the standard deviation of FDs estimated by them is less than 0.01 . Thereafter, by averaging FDs derived from each piece, the overall FD would be estimated. In this study, $L$ was assigned to 11 , and for most of the images, it was seen that a set of 5-10 segments fulfilled the condition of standard deviation. Therefore, depending on the properties of each image, 15-20 points in the logarithmic plot were usually used to estimate FD.

In this study, three different measures of FD were used to investigate changes occurring to the brain WM complexity, including general FD, derived from the overall structure (similar to $\mathrm{FD}_{\mathrm{BG}}$ described in the previous section); boundary (border or surface) FD, derived from WM boundaries (similar to $\mathrm{FD}_{\mathrm{G}}$ ); and skeleton $\mathrm{FD}$, derived from a skeletonized image. The scale of analysis was twofold, global and hemispheric, where the former accounts for the trajectory of changes occurring in whole brain and the latter focuses on the brain hemispheres. The effect of age and sex on the brain complexity is examined in both scales and by means of all three FD measures.

\section{Measuring the Volume}

Tissue probability maps of the WM derived from SPM8, normalized, bias corrected, and roughly aligned to the MNI space (the procedure is described in preprocessing section) were used for a voxel-wise calculation of the volume. As the value of each voxel of a WM probability map (the image after preprocessing) determines the percentage (or probability) of the WM in that voxel, we simply summed up the values of all voxels to calculate the whole WM volume, and a same procedure was repeated for each hemisphere separately.

\section{Modeling the Changes}

We adopted two approaches toward modeling the changes of FD. First, a model selection criterion was used to find the model that best fits the data, but does not indicate statistical significance. This part would help in visualization and understanding the trend of the changes. The second approach involved assessing age- and sex-related changes with statistical significance testing.

\section{Akaike Information Criterion for Model Selection}

To evaluate the changes occurring to the brain FD with aging, we first considered age as a continuous variable and used Akaike Information Criterion (AIC) [22] to find the degree of the polynomial that would best fit the data. AIC uses maximized value of likelihood function to find the best model for a given point set. It, meanwhile, tries to minimize the number of the parameters in the statistical model to reduce the probability of overfitting. In other words, AIC would find the model (viz. polynomial in this study) that maximizes the likelihood with the minimum number of the parameters. For this, we used the polydeg.m function of MATLAB [23]. 


\section{Statistical Analysis}

To examine whether FD measures significantly change with age or sex, we used permutation tests, which are a subgroup of nonparametric tests [24]. According to QQ-plots (Fig. 3) of our data, some features indicated distinct deviations from the normal distribution. Therefore, parametric tests, such as analysis of variance, may not have been applicable and could have yielded an incorrect rate of false-positive results. Permutation tests involved a multiple linear regression with one dependent variable and one or more independent variables. In this test, at first, a primary regression is performed based on the response variable (e.g., FD) and the design matrix (encoding the independent variables, such as age, sex, etc.), so that the primary coefficients are obtained. To obtain the distribution under the null hypothesis, only the independent variable of interest (e.g., age) was randomly permuted and regression repeated based on the new arrangement. Theoretically, this whole procedure should be replicated for any possible permutations. However, as in practice this number of rearrangements would be impossible for large data samples, in this study we used random permutation and replicated the procedure 10,000 times.
It could be perceived that if an FD measure changes significantly with, for example, age, the absolute value of the corresponding linear coefficient in the primary model should be higher than most of the randomized trials. In other words, we expect the coefficients of the random trials to be randomly distributed around 0 , but the coefficient of the features to be significantly more positive/negative. Therefore, the proportion of the randomized trials that yield a higher absolute regression coefficient than the original fit would represent the $p$-value of this test: the higher the proportion, the lower the significance. This method of $p$-value calculation is called a two-sided permutation test.

To perform the permutation test, we first set FDs (each at a time) as dependent variables and age, sex, and the interaction between age and sex as independent or explanatory variables. We also included a column of ones and age $^{2}$ in the design matrix to model the mean effect and control for possible nonlinear effects of the age, respectively. In the next step, we included the WM volume as well, to adjust for possible confounding effects of the volume on FD alterations. Next, we set age as a categorical variable in the design matrix to compare three age cohorts of young, middle-aged, and old and replicated the two aforementioned steps.
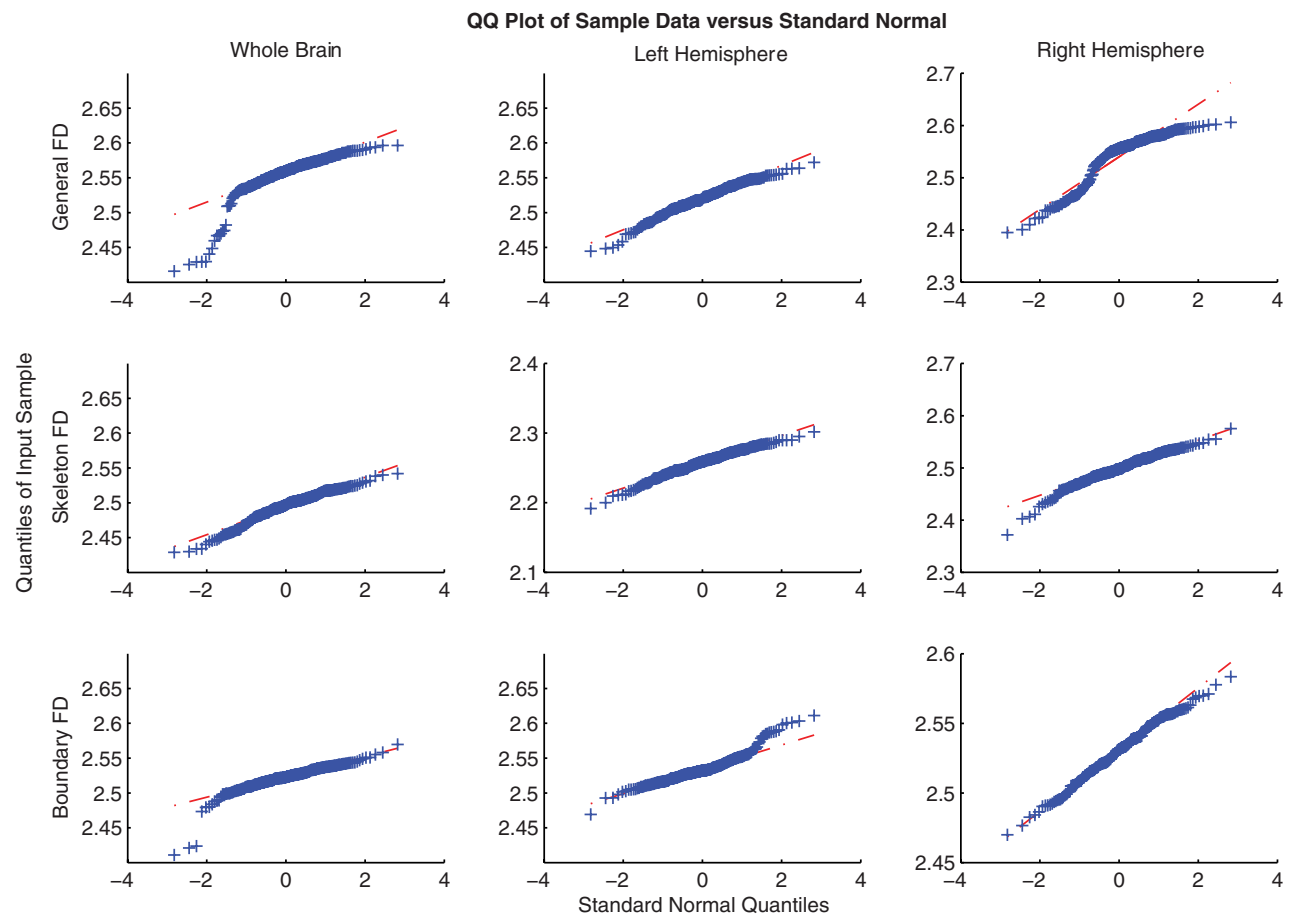

Fig. 3 QQ-plots of the general, skeleton, and boundary fractal dimensions (FDs) of the whole brain and left and right hemisphere. The deviation of the actual point sets from the ideal linear trend indicates the deviation of the data from the normal distribution. In comparison with the ideal normal distribution: top row, general FD of the whole brain was shifted rightward with some outliers; left hemisphere was approximately normal as confirmed by Lilliefors normality test; right hemisphere consisted of a primary distribution shifted rightward and a smaller secondary distribution shifted leftward. Middle row, skeleton FD of the whole brain was shifted rightward; left hemisphere was approximately normal as confirmed by Lilliefors normality test; right hemisphere shifted rightward with some outliers. Bottom row, boundary $F D$ of the whole brain was shifted rightward with some outliers; left hemisphere was shifted leftward with some outliers; right hemisphere was approximately normal as confirmed by Lilliefors normality test 
In addition to investigating the statistical significance, we also measured the Pearson's correlation coefficients $(R)$ between the real data and the regressed model to evaluate the goodness of the fit. To perform the null hypothesis test and check the statistical significance of $R$, a one-sided permutation test was performed assigning the values of the regressed model as fixed variable and permuting the real values of FD.

\section{Results}

Results of the evaluation of the general, skeleton, and boundary FD of the brain WM are reported in the following paragraphs. Differences due to age and sex were observed between the FDs of the whole brain, as well as the left and right hemispheres. Comparisons were made using nine feature sets (three types of FD, for whole brain and the individual hemispheres). Hence, without a correction for multiple comparisons, it is more likely that differences $(p<0.05)$ will be found by chance. To obtain the correct rate of false positives, we used Bonferroni criteria, yielding a significance threshold of 0.0056 .

\section{Akaike Information Criterion}

As illustrated in Fig. 4, for the general FD, AIC detected a quadratic pattern for the whole brain as well as for each hemisphere, increasing from the young to the mid-age and then decreasing to the old. For the skeleton, a quartic (fourth degree) pattern was estimated for the whole brain and the left hemisphere, decreasing from 20 s to the age of 30 , then increasing until the age of 50, decreasing again to the age of 70, and then increasing again. The pattern for the right hemisphere, in contrast, was a quadratic slightly elevating until 40s, reducing with a steeper slope thereafter. For the boundaries, AIC found a zero-degree pattern, indicating that FDs of the boundaries do not change with age.

\section{Permutation Test}

\section{Age as a Continuous Variable}

As indicated in the previous subsection, most FD measures demonstrated nonlinear changing patterns with age. Therefore, for statistical analysis, we first investigated the effect of age, sex, and the interaction of the two, while controlling for nonlinear quadratic effects of the age (age, age $^{2}$, sex, and age $\times$ sex were column vectors of the design matrix). Figure 5a shows the histograms for the coefficients corresponding to age in the regression model during random permutation trials (i.e., the distribution under the null hypothesis), and the red plus sign represents the coefficient without permuting. The further the red sign from the center of the histogram, the lower the $p$-value. All FD measures indicated significant age-related differences for the whole brain as well as in each hemisphere, except for the boundary FD of the left hemisphere.

Figure $5 \mathrm{~b}$ shows the same histograms when sex is assigned as the permutation variable. As could be understood, sex differences were also significant for all the features exclusive of the boundaries of the whole brain. However, looking into more details through Table 1 clarifies that for the border of the left hemisphere, although the regression coefficient given for the age is significantly higher than zero, the regression model that is used to extract this coefficient poorly represents the data, as the $p$-value of the correlation coefficient between the regressed model and the real data is high and $R^{2}$ is rather low. Hereafter, when such conflictions exist, we have used boldface in the tables for emphasis. In these tests, sex is set as a binary variable, with 0 representing men and 1 representing women. It could be understood from the diagrams that sex coefficient in the primary model is negative, indicating that men showed higher FD values than women. The effect of age $\times$ sex, as illustrated in Fig. 6 , was specifically evident in the hemispheric analysis, with men undergoing a sharper reduction in the complexity of the skeleton and boundaries of the left hemisphere as well as the general and skeleton of the right. Women, in contrast, displayed an increase in the boundary FD of the right hemisphere, whereas the pattern was again decreasing for men.

Table 1 elaborates the results of the analyses including primary regression coefficient for all independent variables, the significance of the differences, as well as the significance of the fit. Columns 2-5 contain the primary regression coefficients corresponding to the age, $\mathrm{age}^{2}$, sex, and age $\times$ sex, respectively, with $b_{0}$ showing the bias value of that model. Columns $6-8$ include $p$-values to evaluate the statistical significance of the age, sex, and age- $\times$ sex-related changes of the features, and columns 9-10 contain correlation coefficients and $p$-values to observe the goodness of the regressed model.

Adjusting for the Volume Effect: In the next step, we added the WM volume to the design matrix. When investigating FDs of the whole brain WM, the global WM volume was taken into account, whereas in the hemispheric analysis, the volume of the corresponding hemisphere was controlled for. As detailed in Table 2, all scales of the general and skeleton FD changed significantly with age, but for the boundaries, only the whole brain indicated significant age-related alterations.

For the sex differences, some obvious alterations in the pattern appeared compared with the former analysis. Unlike before, higher complexities were found for the women except for all the features of the right hemisphere and 

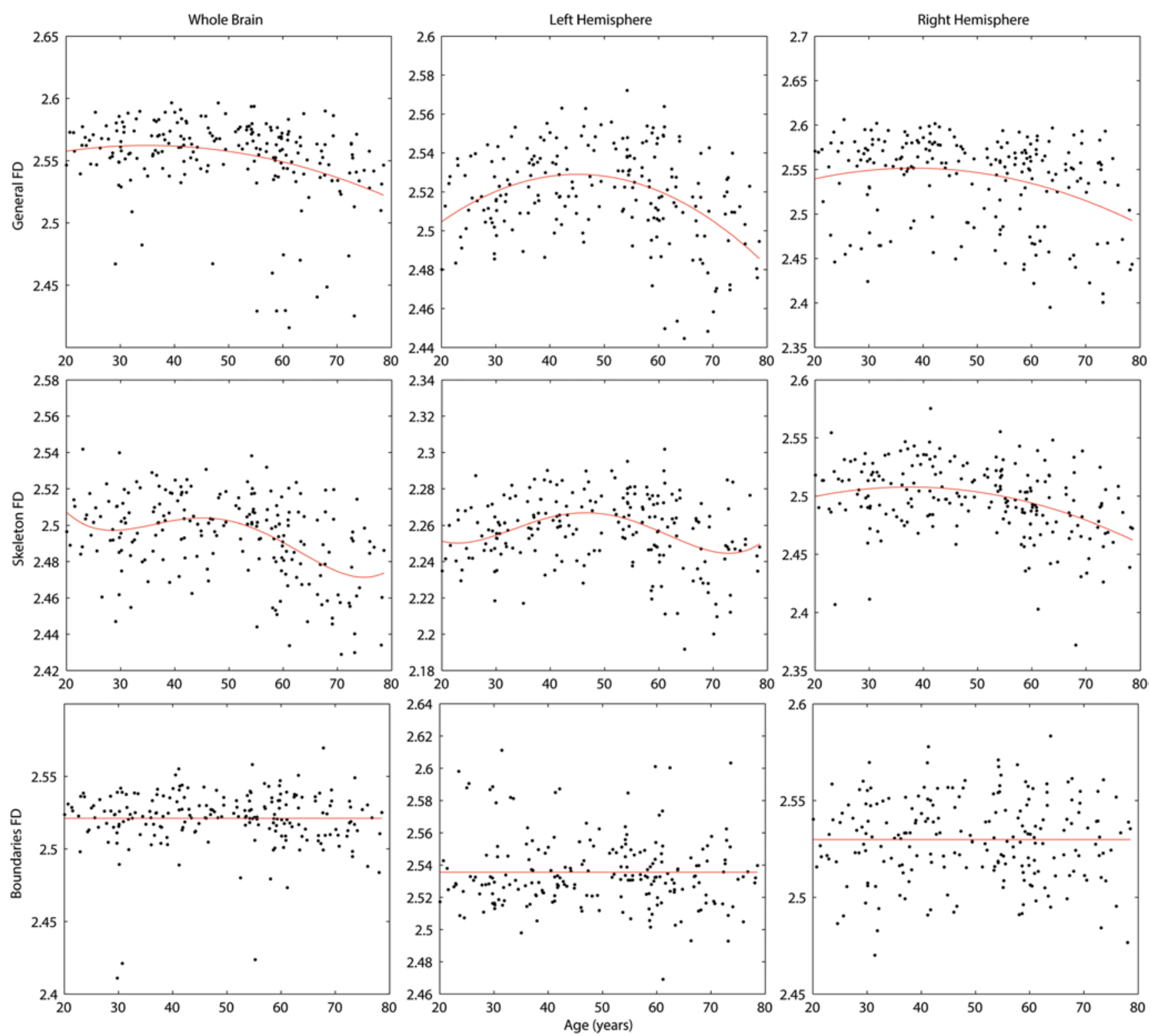

Fig. 4 Patterns of changes of the general, skeleton, and boundary fractal dimensions $(F D \mathrm{~s})$ of the whole brain and left and right hemisphere with age (years). The black point sets represent the actual data points; the solid fit curves are the polynomials as detected by Akaike Information Criterion model selection and fitted by polyfit.m function in MATLAB boundary FD of the left (for this one, the regressed model was poor). However, only in the boundary of the whole brain and general structure of the left hemisphere, the values reached significance. One other important point was that the right hemisphere remained significantly more complex in men. The effect of age $\times$ sex disclosed significant changes only for the hemispheric analysis except for the general FD of the left hemisphere.

To further evaluate the correlations between the volume and FD measures, we calculated correlation coefficients for the whole brain. General, skeleton, and boundary FD, respectively, disclosed $0.48,0.558$, and 0.385 positive correlation coefficients (all $p$-values $<10^{-4}$ ) with the WM volume.

\section{Categorical Comparisons}

In categorical age analysis, we classified all subjects into three groups of young, mid-age, and old and compared the age cohorts. As expected, patterns were consistent with those from the continuous age evaluations. As elaborated in Table 3, age-group effects were significant for all FD measures, with $p<0.0002$, and the sex effect was significant for all but the whole brain boundaries. Age and sex interaction 

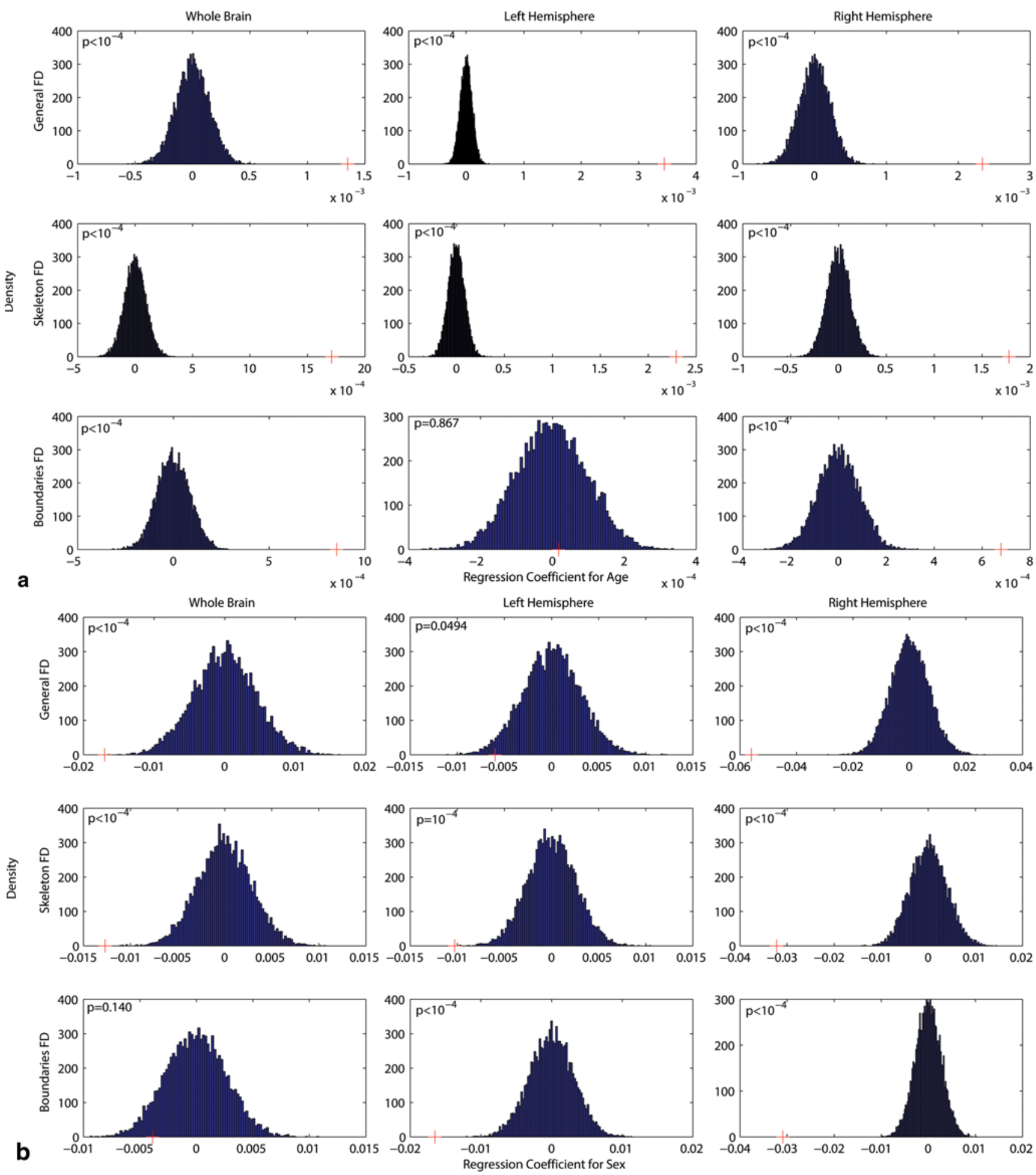

Fig. 5 Results of permutation test when assigning age $\mathbf{a}$ and $\operatorname{sex} \mathbf{b}$ as the randomly permuted variable ( $F D$ fractal dimension). The histograms indicate the regression coefficients corresponding to the variable of interest during randomization trials, and the red plus sign indicates the primary coefficient corresponding to that variable (before permutation process). The further the plus sign from the histogram, the more significant the changes of the feature of interest with the variable of interest (i.e., age or sex). $p$-Values are also displayed at the top left corner of each plot 

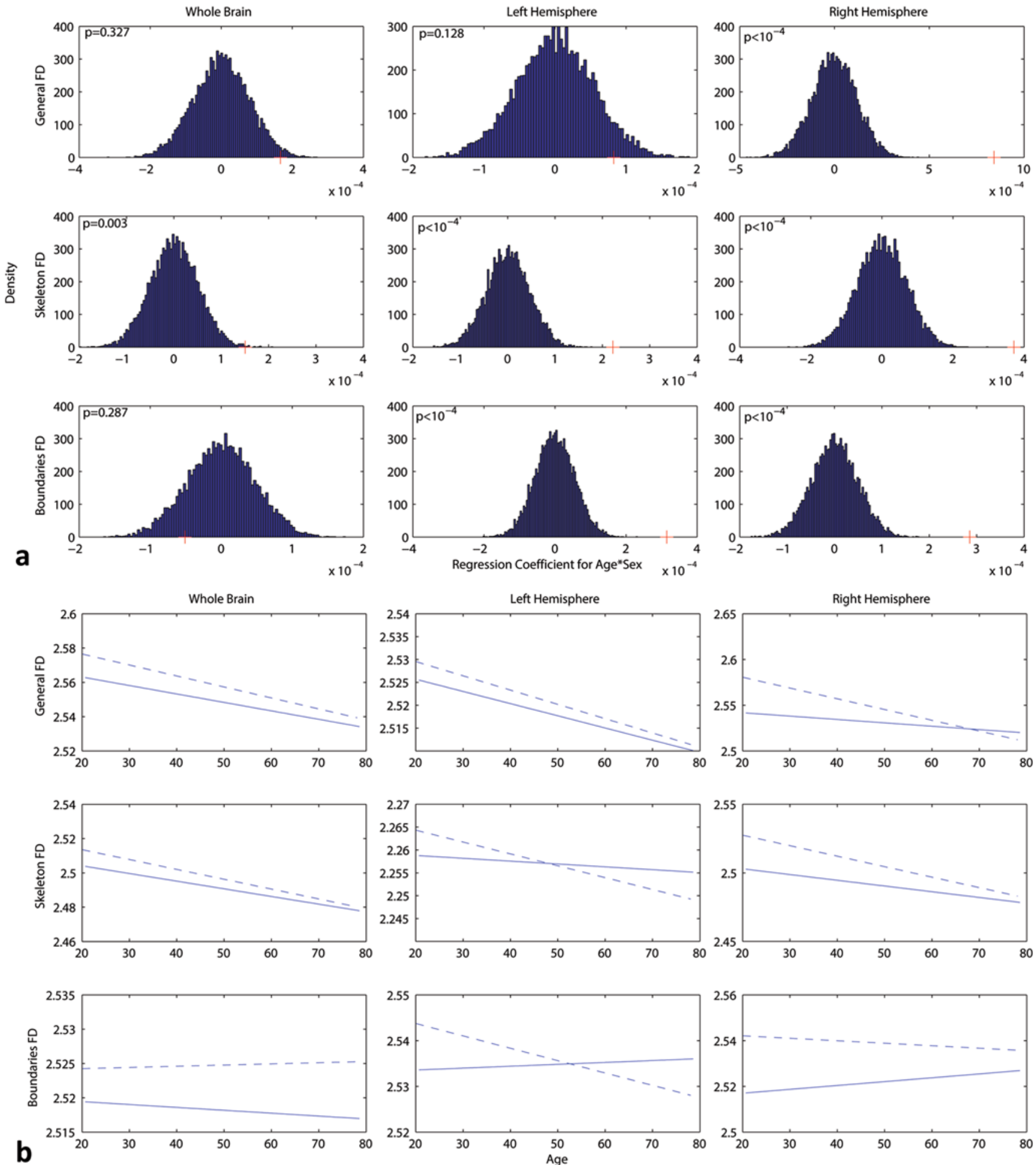

Fig. 6 a Results of the permutation test when assigning age $\times$ sex as the randomly permuted variable. The effect of age $\times$ sex interaction was significant on the general fractal dimension (FD) of the right hemisphere, skeleton FD of all scales, and boundary FD of the two hemispheres. b Comparison of the linear trends of age-related changes of the FDs of the global and hemispheric WM between men (dashed curves) and women (solid curves). Men's brains undergo more intense changes with aging and the slope of the trend is different between the two genders in the boundary FD 
Table 1 Results of the statistical analysis while treating age as a continuous variable, before controlling for the volume effect

\begin{tabular}{|c|c|c|c|c|c|c|c|c|c|c|}
\hline & \multicolumn{5}{|c|}{ Regression coefficients } & \multicolumn{3}{|c|}{ Change significance ( $p$-value) } & \multicolumn{2}{|c|}{ Regression significance } \\
\hline & $\mathrm{b}_{0}$ & Age & $\mathrm{Age}^{2}$ & Sex & $\overline{\text { Age } \times \operatorname{sex}}$ & Age & Sex & Age $\times$ sex & $\overline{R^{2}}$ & $p$-value \\
\hline \multicolumn{11}{|c|}{$\overline{\text { General FD }}$} \\
\hline Whole & 2.546 & 0.0014 & $-2.05 \times 10^{-5}$ & -0.017 & 0.00017 & $<10^{-4}$ & $<10^{-4}$ & 0.327 & 0.116 & $<10^{-4}$ \\
\hline Left & 2.453 & 0.0034 & $-3.86 \times 10^{-5}$ & -0.006 & $8.3 \times 10^{-5}$ & $<10^{-4}$ & $<10^{-4}$ & 0.128 & 0.176 & $<10^{-4}$ \\
\hline Right & 2.527 & 0.0023 & $-3.61 \times 10^{-5}$ & -0.056 & 0.00084 & $<10^{-4}$ & $<10^{-4}$ & $<10^{-4}$ & 0.127 & $<10^{-4}$ \\
\hline \multicolumn{11}{|c|}{ Skeleton FD } \\
\hline Whole & 2.475 & 0.0017 & $-2.35 \times 10^{-5}$ & -0.013 & 0.00015 & $<10^{-4}$ & $<10^{-4}$ & 0.003 & 0.202 & $<10^{-4}$ \\
\hline Left & 2.214 & 0.0023 & $-2.62 \times 10^{-5}$ & -0.010 & 0.00022 & $<10^{-4}$ & $<10^{-4}$ & $<10^{-4}$ & 0.115 & $<10^{-4}$ \\
\hline Right & 2.487 & 0.0018 & $-2.61 \times 10^{-5}$ & -0.032 & 0.00037 & $<10^{-4}$ & $<10^{-4}$ & $<10^{-4}$ & 0.205 & $<10^{-4}$ \\
\hline \multicolumn{11}{|c|}{ Boundary FD } \\
\hline Whole & 2.506 & 0.0009 & $-8.59 \times 10^{-6}$ & -0.004 & $-5.1 \times 10^{-5}$ & $<10^{-4}$ & 0.14 & 0.287 & 0.040 & 0.003 \\
\hline Left & 2.543 & $1.67 \times 10^{-5}$ & $-2.95 \times 10^{-6}$ & -0.016 & 0.00032 & 0.87 & $<10^{-4}$ & $<10^{-4}$ & 0.018 & 0.05 \\
\hline Right & 2.527 & 0.0007 & $-8.09 \times 10^{-6}$ & -0.031 & 0.00029 & $<10^{-4}$ & $<10^{-4}$ & $<10^{-4}$ & 0.179 & $<10^{-4}$ \\
\hline
\end{tabular}

$F D$ fractal dimension

Table 2 Results of statistical analysis while considering age as a continuous variable, after controlling for the volume effect

\begin{tabular}{|c|c|c|c|c|c|c|c|c|c|c|c|}
\hline & \multicolumn{6}{|c|}{ Regression coefficients } & \multicolumn{3}{|c|}{ Change significance ( $p$-value) } & \multicolumn{2}{|c|}{ Regression significance } \\
\hline & $\mathrm{b}_{0}$ & Age & $\mathrm{Age}^{2}$ & Sex & Volume & Age $\times$ sex & Age & Sex & $\overline{\text { Age } \times \operatorname{sex}}$ & $\overline{R^{2}}$ & $p$-value \\
\hline \multicolumn{12}{|c|}{$\overline{\text { General FD }}$} \\
\hline Whole & 2.416 & 0.0005 & $-8.6 \times 10^{-6}$ & 0.0067 & $2.7 \times 10^{-7}$ & $6.27 \times 10^{-5}$ & $<10^{-4}$ & 0.096 & 0.377 & 0.278 & $<10^{-4}$ \\
\hline Left & 2.334 & 0.0026 & $-2.8 \times 10^{-5}$ & 0.0154 & $5.1 \times 10^{-7}$ & $-8.85 \times 10^{-6}$ & $<10^{-4}$ & $<10^{-4}$ & 0.836 & 0.446 & $<10^{-4}$ \\
\hline Right & 2.413 & 0.0015 & $-2.6 \times 10^{-5}$ & -0.035 & $4.7 \times 10^{-7}$ & 0.0007 & $<10^{-4}$ & $<10^{-4}$ & $<10^{-4}$ & 0.183 & $<10^{-4}$ \\
\hline \multicolumn{12}{|c|}{ Skeleton FD } \\
\hline Whole & 2.373 & 0.0010 & $-1.4 \times 10^{-5}$ & 0.006 & $2.1 \times 10^{-7}$ & $6.89 \times 10^{-5}$ & $<10^{-4}$ & 0.018 & 0.115 & 0.416 & $<10^{-4}$ \\
\hline Left & 2.142 & 0.0018 & $-1.97 \times 10^{-5}$ & 0.0024 & $3 \times 10^{-7}$ & 0.00016 & $<10^{-4}$ & 0.288 & $<10^{-4}$ & 0.259 & $<10^{-4}$ \\
\hline Right & 2.329 & 0.0007 & $-1.18 \times 10^{-5}$ & -0.003 & $6.5 \times 10^{-7}$ & 0.00024 & $<10^{-4}$ & 0.3 & $<10^{-4}$ & 0.496 & $<10^{-4}$ \\
\hline \multicolumn{12}{|c|}{ Boundary FD } \\
\hline Whole & 2.441 & 0.0004 & $-2.68 \times 10^{-6}$ & 0.008 & $1.4 \times 10^{-7}$ & -0.0001 & $<10^{-4}$ & 0.0007 & 0.022 & 0.159 & $<10^{-4}$ \\
\hline Left & 2.492 & -0.0003 & $1.72 \times 10^{-6}$ & -0.007 & $2.2 \times 10^{-7}$ & 0.00028 & $<10^{-4}$ & 0.012 & $<10^{-4}$ & 0.075 & 0.0001 \\
\hline Right & 2.434 & $3.7 \times 10^{-5}$ & $3.39 \times 10^{-7}$ & -0.014 & $3.8 \times 10^{-7}$ & 0.00021 & 0.62 & $<10^{-4}$ & $<10^{-4}$ & 0.385 & $<10^{-4}$ \\
\hline
\end{tabular}

$F D$ fractal dimension

Table 3 Categorical analysis of the age cohorts

\begin{tabular}{|c|c|c|c|c|c|c|c|c|c|}
\hline & \multirow{2}{*}{\multicolumn{3}{|c|}{ Age-group }} & \multicolumn{6}{|c|}{$P$-value from the permutation test } \\
\hline & & & & \multicolumn{3}{|c|}{ Before volume control } & \multicolumn{3}{|c|}{ After volume control } \\
\hline & Young & Mid-age & Old & Age & Sex & Age $\times$ sex & Age & Sex & Age $\times$ sex \\
\hline \multicolumn{10}{|c|}{ General FD } \\
\hline Whole & $2.56 \pm 0.023$ & $2.559 \pm 0.03$ & $2.535 \pm 0.04$ & $<10^{-4}$ & $<10^{-4}$ & 0.0017 & $<10^{-4}$ & 0.4392 & 0.0648 \\
\hline Left & $2.52 \pm 0.018$ & $2.527 \pm 0.02$ & $2.506 \pm 0.028$ & $<10^{-4}$ & 0.0016 & 0.014 & $<10^{-4}$ & $<10^{-4}$ & 0.3889 \\
\hline Right & $2.548 \pm 0.045$ & $2.545 \pm 0.042$ & $2.518 \pm 0.057$ & $<10^{-4}$ & $<10^{-4}$ & $<10^{-4}$ & $<10^{-4}$ & 0.0004 & 0.0001 \\
\hline \multicolumn{10}{|c|}{ Skeleton FD } \\
\hline Whole & $2.5 \pm 0.019$ & $2.499 \pm 0.019$ & $2.48 \pm 0.025$ & $<10^{-4}$ & $<10^{-4}$ & 0.0044 & $<10^{-4}$ & 0.0079 & 0.1795 \\
\hline Left & $2.257 \pm 0.016$ & $2.263 \pm 0.016$ & $2.249 \pm 0.024$ & $<10^{-4}$ & $<10^{-4}$ & $<10^{-4}$ & $<10^{-4}$ & 0.6854 & $<10^{-4}$ \\
\hline Right & $2.504 \pm 0.028$ & $2.503 \pm 0.025$ & $2.482 \pm 0.033$ & $<10^{-4}$ & $<10^{-4}$ & 0.0002 & $<10^{-4}$ & 0.1959 & 0.0593 \\
\hline \multicolumn{10}{|c|}{ Boundary FD } \\
\hline Whole & $2.519 \pm 0.022$ & $2.525 \pm 0.018$ & $2.519 \pm 0.017$ & $<10^{-4}$ & 0.1844 & 0.1298 & $<10^{-4}$ & 0.0001 & 0.0049 \\
\hline Left & $2.536 \pm 0.025$ & $2.537 \pm 0.019$ & $2.532 \pm 0.023$ & 0.0002 & $<10^{-4}$ & $<10^{-4}$ & 0.7605 & 0.0943 & $<10^{-4}$ \\
\hline Right & $2.528 \pm 0.02$ & $2.533 \pm 0.021$ & $2.528 \pm 0.022$ & $<10^{-4}$ & $<10^{-4}$ & 0.0001 & $<10^{-4}$ & $<10^{-4}$ & 0.0072 \\
\hline
\end{tabular}

$F D$ fractal dimension 
was meaningful for all the measures (all $p$-values $<0.001$ ), exclusive of the general structure of the whole brain and boundaries of the left hemisphere. The $p$-values for the border of the left hemisphere were considered invalid due to the poor regressed model. After controlling for the volume effect, associations with most features remained significant for age, sex, or their interaction (Table 3).

In Table 3, columns 1-3 represent mean FD \pm standard deviation for the young, mid-age, and old, respectively; columns 4-5 contain $p$-values corresponding to the main effects of age and sex; and column 6 includes $p$-values of age $\times$ sex interaction, all before controlling for the volume effect. Columns 7-9 represent similar corresponding $p$-values after adjusting for the volume effect.

\section{Discussion}

In this study, age-related changes occurring in the complexity of the brain WM of normal adults were investigated by means of FD, with higher FD showing a more complex structure. The changes were evaluated with two different approaches: a model selection criterion and a nonparametric statistical analysis. For model selection, AIC was used to determine the degree of the polynomial that best fits the data. AIC selected an inverted U-shape quadratic pattern of age-related changes in the general FD of the whole brain, as well as for each hemisphere. For the skeleton, the pattern was a quartic for the whole brain and the left hemisphere and a quadratic for the right. For the boundaries, AIC selected a constant-with-age pattern. These results indicated an increasing trend for FDs of the general structure and the skeleton from young to mid-age, then a decreasing pattern with a sharper slope from mid-age to the old. Permutation tests disclosed significant age-related changes in the whole brain, as well as in each hemisphere, by means of all the three FD measures. However, evaluating the boundaries, no significant change was found for the right hemisphere and no "good" fit for the whole brain or left hemisphere. After controlling for volume, the overall patterns of the general and skeleton FDs did not change notably.

To our knowledge, there is only one previous study that comprehensively investigated age- and sex-related changes of FD of the brain WM in normal adults [7]. The authors compared two groups of young (17-35 years) and old (7280 years) with total 36 subjects, extracting 3D FD from the general, skeleton, and boundaries of the whole brain as well as each hemisphere. Evaluating the general and skeleton structures, they found that FD decreases in elderly, which was significant for both features at a global scale but only for the left hemisphere of skeleton at hemispheric scale. The patterns found in this study, as displayed in Fig. 4, suggest that the differences between people younger than 35 years and those older than 70 years are apparent for all these six features/scales, except for the left hemisphere of the skeleton. Therefore, it seems that the general trends in our results are broadly similar to theirs. In the boundaries, they reported no notable change from the young to the old group, which matches what we have found. They also investigated sex effects and reported higher FDs in men, with significance for the three measures in the whole brain and for the skeleton and surface of the right hemisphere. They reported no age $\times$ sex interaction. Overall, the results for the global analyses are approximately matching between the two studies, but in the hemispheric scale, some incongruities are visible. These differences between our findings and those of the previous study might have been raised from various sources, most importantly the characteristics of the populations under investigation.

In our study, the gradual-increase-sharper-decrease patterns found for the general and skeleton structures may be related to those of the previous volumetric studies, which suggested that WM loss starts in late mid-age and thereafter progresses rapidly (for a review, see [3]). To check the relationships between our results and those of the volumetric analyses, we calculated the correlation coefficients between the whole brain WM volume and FD measures and found positive correlations for all the three FDs varying from 0.39 to 0.56 as elaborated in "Results" section. Therefore, our age-related results are in line with the previous volumetric studies and demonstrate that larger WM volume is associated with more complex structure. The results also indicate that although volume and complexity are correlated, but since this correlation is not $100 \%$, and more importantly, since even after adjusting for the volume effects the agerelated variations remain meaningful, some other structural changes may also contribute toward decreasing FD.

An emerging concept in studying the complexity and connectivity of the brain structural and functional networks is the small-world characteristic. Previous studies demonstrate that small-world metrics (such as clustering coefficient and path length) are mutually correlated to the network scale-freeness as described by fractality [25]. Small-world properties, together with fractality, provide an optimal organization for the brain architecture that lets it convey the most information with the least wiring, and this makes these two characteristics important in terms of evolution [26]. A recent study [27] evaluating the small-world characteristics of the structural networks of the brain in normal aging found an inverted U-shape age-related pattern of changes for the integrated global efficacy of the networks. As shown by that study, the efficacy of the global networks of the brain increases from the young (18-40 years old) to mid-age (4160 years old), then decreases in the elderly (61-80 years old), where the young group's global efficacy was the least of all. The authors linked these findings to the changes in the 
global WM and GM structure and concluded that the "maturity" of the brain increases from the young to the mid-age, and then a "degeneration" process starts and continues to the elderly. Although not directly measured from the WM, these findings are compatible with the trends of our results for the WM general and interior structure, where the pattern was also an inverted $U$ shape that peaked at late 40 s. Therefore, our results could relate that FD of structural brain images, which is a single number that is calculated with a fast and relatively convenient $\mathrm{BC}$ algorithm, could give us useful information about the characteristics of the complex structural and consequently functional [28] networks of the brain.

In another recent study [29], Fernández et al. also reported an inverted U-shape curvilinear pattern of the changes for the complexity of the brain Magnetoencephalography (MEG) oscillations with age, reaching its peak at 60 s. According to them, power spectral measures of the brain neurophysiological signals (i.e., Electroencephalography (EEG) and MEG) are more correlated with GM structure, and complexity measures are more correlated with WM structure. Hence, the quadratic pattern found in our study matches the trend that is reported by them and could support the existence of an interaction between the WM structure and the brain functional complexity. This interpretation is generally in accordance with the prevalent claim of strong interactions between the brain function and structure [28, 30]. Moreover, the comparison between the results of the two studies might suggest that age-related changes start from the brain structure and later appear in the function. However, a combined functional-structural study on a single dataset is required to investigate this suggestion.

Furthermore, an earlier study by Fernández et al. [31] found a positive correlation between WM structural integrity and the brain's oscillatory complexity. Basing their discussion on the prevalent notion that the complexity represents the number of the independent oscillators of the brain [32], they argued that changes in the brain structures that play a role in the formation of the oscillators should account for the quadratic results obtained from their research. According to them, WM myelination and demyelination during development and aging could be the process that accounts for the changes in the circuitry design of the brain oscillators and hence the corresponding oscillations. Besides, some previous studies have shown that myelination continues during childhood, adolescence, and early adulthood (up to the beginning of 30s) and may also continue further [33, 34]. Moreover, recently many aging studies have reported decreased integrity of WM due to demyelination of associative fibers and/or axonal cell membrane loss, and consequently less connected neuronal networks in the elderly $[4,35]$. Two of the most commonly used measures, which indicate decline of WM integrity in elderly, are decreased FA extracted from DTIs [3-5] and appearance of WMH in T2-weighted images [3, 36]. Another common measure for quantification of the demyelination from brain images has been the MTR of the WM, which is shown to demonstrate a nonsymmetrical inverse U-shape pattern with a slight increase of MTR up until mid-40s and a sharper decrease thereafter [37]. Overall, it is possible that this myelination/demyelination procedure results in generation/loss of some WM fiber crossing and bifurcations [7], increase/reduce the connectivity, and change the complexity of WM correspondingly. These findings could explain the curvilinear patterns that are found in this study, connecting WM structural complexity to the WM integrity and myelination/demyelination during evolution and aging. Comparative studies between WM demyelination quantifiers and FD analysis could shed light on this hypothesis.

Sex dissimilarities were also examined by means of the three FD features (general, skeleton, and boundary FD), and, before correcting for the volume effect, men displayed significantly higher FDs (except for two features elaborated in Table 1). After correcting for volume effects, the patterns were reversed in the whole brain and the left hemisphere of the general and interior structure, viz. women exhibited higher complexities in most of the features, including all the three FD measures from the whole brain. According to the findings of [31], these results might also be related to the previously reported higher complexities of EEG and MEG signals in women [29, 38]. However, it is worthwhile noting that among the five of nine features that women indicated higher FDs for, the difference was significant only for the boundary FD of the whole brain and general FD of the left hemisphere. Thus, our results for the pre-volume-control are in line with the previously reported higher WM volume [39] and FD [7] in men. This is while our results for postvolume-control indicates that if we remove the effect of the size on the FD, the general structure of the left hemisphere is more complex in women and the general structure of the right hemisphere is more complex in men. A recent study [40] that compared small-world characteristics of the brain networks between men and women found higher clustering coefficient in men's right hemisphere and in women's left hemisphere. They concluded that the left hemisphere, mostly associated with language tasks, is more complex/ developed in women, whereas the right hemisphere, which is more specified for visuotemporal tasks, is more developed in men. This reasoning is also in alignment with previous behavioral studies [41]. According to the aforementioned interrelationships between small-world properties and fractality, our results characterizing the scale-freeness of brain networks are aligned with the sex differences in the brain lateralization as obtained from small-world analysis and might account for gender differences in task performances using a similar logic. This positive correlation between WM 
complexity and cognitive ability is also stated in a recent research [15]. Another finding of our study with respect to sex differentiation after controlling for volume was that women have a more complex WM outline. Although these results might be related to those of the former studies that found more complex brain cortex [42] in women, we should make this connection very cautiously, as the boundary FD in this study is calculated from all the so-called "gray" voxels of the image as described in "Materials and Methods" section. As could be understood, these voxels comprise both GM adjacent border and ventricle adjacent border of WM. Separating these two in future studies might yield a clearer view. We also evaluated age $\times$ sex interaction effects and found significance mainly in the hemispheric scale analysis, with men undergoing steeper changes with age.

\section{Conclusion}

This study is the first to examine differences in the complexity of WM by means of FD, using a large dataset that covers a wide age range of adult lifespan, treating age as a continuous variable, and also comparing age cohorts. We found positive correlations between FD and volume, and our results show similarities with those that have investigated small-world properties of brain networks, as well as those that observed functional complexity and WM integrity. Although it is not clear to which of these characteristics FD mostly attaches, similar trends suggest that FD could yield a highly compact description of the structural changes, and might also inform us about functional and cognitive alterations of the brain. Sensitivity of FD to age and sex variations as described in this study, as well as its ability to diagnose neurologic/neuropsychiatric disease from the brain images as indicated in some of the previous studies $[11,12,43]$, could suggest this feature as a useful tool for the clinical studies. Future studies directly comparing FD with other structural features, functional characteristics, and behavioral outcomes could be expected to further clarify the clinical interpretation of this feature.

Acknowledgments The Wellcome Trust Centre for Neuroimaging is supported by core funding from the Wellcome Trust [grant number 091593/Z/10/Z].

Conflict of Interest On behalf of all authors, the corresponding author states that there is no conflict of interest.

Open Access This article is distributed under the terms of the Creative Commons Attribution Noncommercial License which permits any noncommercial use, distribution, and reproduction in any medium, provided the original author(s) and the source are credited.

\section{References}

1. Lemaître H, Crivello F, Grassiot B, Alpérovitch A, Tzourio C, Mazoyer B. Age- and sex-related effects on the neuroanatomy of healthy elderly. Neuroimage. 2005;26(3):900-11.

2. Good CD, Johnsrude IS, Ashburner J, Henson RN, Friston KJ, Frackowiak RS. A voxel-based morphometric study of ageing in 465 normal adult human brains. Neuroimage. 2001;14:21-36.

3. Gunning-Dixon FM, Brickman AM, Cheng JC., Alexopoulos GS. Aging of cerebral white matter: a review of MRI findings. Int $\mathrm{J}$ Geriatr Psychiatry. 2009;24(2):109-17.

4. Kochunov P, Thompson PM, Lancaster JL, Bartzokis G, Smith $\mathrm{S}$, Coyle $\mathrm{T}$, et al. Relationship between white matter fractional anisotropy and other indices of cerebral health in normal aging: tract-based spatial statistics study of aging. Neuroimage. 2007;35(2):478-87.

5. Grieve SM, Williams LM, Paul RH, Clark CR, Gordon E. Cognitive aging, executive function, and fractional anisotropy: a diffusion tensor MR imaging study. AJNR Am J Neuroradiol. 2007;28(2):226-35.

6. Brickman AM, Habeck C, Zarahn E, Flynn J, Stern Y. Structural MRI covariance patterns associated with normal aging and neuropsychological functioning. Neurobiol Aging. 2007;28(2):284-95.

7. Zhang L, Dean D, Liu JZ, Sahgal V, Wang X, Yue GH. Quantifying degeneration of white matter in normal aging using fractal dimension. Neurobiol Aging. 2007;28:1543-55.

8. Zhang L, Liu JZ, Dean D, Sahgal V, Yue GH. A three-dimensional fractal analysis method for quantifying white matter structure in human brain. J Neurosci Methods. 2006;150(2):242-53.

9. Im K, Lee JM, Yoon U, Shin YW, Hong SB, Kim IY, et al. Fractal dimension in human cortical surface: multiple regression analysis with cortical thickness, sulcal depth, and folding area. Hum Brain Mapp. 2006;27(12):994-1003.

10. Mandelbrot B. How long is the coast of Britain? Statistical self-similarity and fractional dimension. Science. 1967;156(3775):636-8.

11. Esteban FJ, Sepulcre J, de Mendizábal NV, Goñi J, Navas J, de Miras JR, et al. Fractal dimension and white matter changes in multiple sclerosis. Neuroimage. 2007;36(3):543-9.

12. King RD, Brown B, Hwang M, Jeon T, George AT. Alzheimer's Disease Neuroimaging Initiative. Fractal dimension analysis of the cortical ribbon in mild Alzheimer's disease. Neuroimage. 2010;53(2):471-9.

13. Bojić T, Vuckovic A, Kalauzi A. Modeling EEG fractal dimension changes in wake and drowsy states in humans - a preliminary study. J Theor Biol. 2010;262(2):214-22.

14. Lopes R, Betrouni N. Fractal and multifractal analysis: A review. Med Image Anal. 2009;13(4):634-49.

15. Mustafa N, Ahearn TS, Waiter GD, Murray AD, Whalley LJ, Staff RT. Brain structural complexity and life course cognitive change. Neuroimage. 2012;61(3):694-701.

16. Biomedical Image Analysis Group, Imperial College London. brain-development.org@imperial college. http://brain-development.org/.

17. Ashburner J, Friston KJ. Unified segmentation. Neuroimage. 2005;26(3):839-51.

18. Ashburner, J. A fast diffeomorphic image registration algorithm. Neuroimage. 2007;38(1):95-113.

19. Klein A, Hirsch J. Mindboggle: a scatterbrained approach to automate brain labeling. Neuroimage. 2005;24(2):261-80.

20. Wang T, Basu A. A note on 'A fully parallel $3 \mathrm{D}$ thinning algorithm and its applications'. Pattern Recognit Lett. 2007;28(4):501-6.

21. Russel D, Hanson J, Ott E. Dimension of strange attractors. Phys Rev Lett. 1980;45(14):1175-8.

22. Akaike H. A new look at the statistical model identification. IEEE Transactions on Automatic Control. 1974;19(6):716-23. 
23. Garcia D. Department of radiology, CRCHUM, University of Montreal Hospital, "BioméCardio". 1. 2010. http://www. biomecardio.com/matlab/polydeg.html. Accessed 2 Oct 2013.

24. Edgington ES. Randomization tests. 3rd edn. New York: MarcelDekker; 1995.

25. Sporns O. Small-world connectivity, motif composition, and complexity of fractal neuronal connections. BioSystems. 2006;85, $55-64$.

26. Tian L, Wang J, Yan C, He Y. Hemisphere- and gender-related differences in small-world brain networks: a resting-state functional MRI study. Neuroimage. 2011;54(1):191-202.

27. Wu K, Taki Y, Sato K, Kinomura S, Goto R, Okada K, et al. Age-related changes in topological organization of structural brain networks in healthy individuals. Hum Brain Mapp. 2012;33(3):552-68.

28. Gallos LK, Sigman M, Makse HA. The conundrum of functional brain networks: small-world efficiency or fractal modularity. Front Physiol. 2012;3:123.

29. Fernández A, Zuluaga P, Abásolo D, Gómez C, Serra A, Méndez MA, et al. Brain oscillatory complexity across the life span. Clin Neurophysiol. 2012;123(11):2154-62.

30. Rubinov M, Sporns O. Complex network measures of brain connectivity: uses and interpretations. Neuroimage. 2010;52(3):1059-69.

31. Fernández A, Ríos-Lago M, Abásolo D, Hornero R, Alvarez-Linera $\mathrm{J}$, Paul N, et al. The correlation between white-matter microstructure and the complexity of spontaneous brain activity: a difussion tensor imaging-MEG study. Neuroimage. 2011;57(4):1300-7.

32. Lutzenberger W, Preissl H, Pulvermüller F. Fractal dimension of electroencephalographic time series and underlying brain processes. Biol Cybern. 1995;73(5):477-82.

33. Miller DJ, Duka T, Stimpson CD, Schapiro SJ, Baze WB, McArthur MJ, et al. Prolonged myelination in human neocortical evolution. Proc Natl Acad Sci U S A. 2012;109(41):16480-5.
34. Yakovlev PI, Lecours AR. The myelogenetic cycles of regional maturation of the brain. Regional development of the brain in early life. Oxford: Blackwell Science; 1967. pp. 3-70.

35. Bartzokis G, Cummings JL, Sultzer D, Henderson VW, Nuechterlein $\mathrm{KH}$, Mintz J. White matter structural integrity in healthy aging adults and patients with Alzheimer disease: a magnetic resonance imaging study. Arch Neurol. 2003;60(3):393-8.

36. Söderlund $H$, Nyberg L, Adolfsson R, Nilsson LG, Launer LJ. High prevalence of white matter hyperintensities in normal aging: relation to blood pressure and cognition. Cortex. 2003;39(4-5):1093-105.

37. Ge Y, Grossman RI, Babb JS, Rabin ML, Mannon LJ, Kolson DL. Age-related total gray matter and white matter changes in normal adult brain. Part II: quantitative magnetization transfer ratio histogram analysis. AJNR Am J Neuroradiol. 2002;23(8):1334-41.

38. Ahmadi K, Ahmadlou M, Rezazade M, Azad-Marzabadi E, Sajedi F. Brain activity of women is more fractal than men. Neurosci Lett. 2013;535:7-11.

39. Gur RC, Turetsky BI, Matsui M, Yan M, Bilker W, Hughett P, et al. Sex differences in brain gray and white matter in healthy young adults: correlations with cognitive performance. J Neurosci. 1999;19(10):4065-72.

40. Tian L, Wang J, Yan C, He Y. Hemisphere- and gender-related differences in small-world brain networks: a resting-state functional MRI study. Neuroimage. 2011;54(1):191-202.

41. Hamilton C. Cognition and sex differences. New York: Palgrave Macmillan; 2008.

42. Luders E, Narr KL, Thompson PM, Rex DE, Jancke L, Steinmetz $\mathrm{H}$, et al. Gender differences in cortical complexity. Nat Neurosci. 2004;7(8):799-800.

43. Ha TH, Yoon U, Lee KJ, Shin YW, Lee JM, Kim IY, et al. Fractal dimension of cerebral cortical surface in schizophrenia and obsessive-compulsive disorder. Neurosci Lett. 2005;384(1-2):172-6. 